\title{
Implementation of Karaoke Machine on the DSK6713 Processor
}

\author{
Nirmal R Bhalani \\ M.Tech. (Pursuing) \\ E.C. Dept., SSSIST \\ Sehore, India
}

\author{
Jaikaran Singh \\ Head of Department \\ E.C. Dept., SSSIST \\ Sehore, India
}

\author{
Mukesh Tiwari \\ Dean Academics \\ SSSIST \\ Sehore, India
}

\begin{abstract}
In this paper we present an implementation of karaoke machine for vocal. Karaoke is a form of entertainment in which users sing a song along with recorded music using microphone. In our first step we have successfully designed a karaoke machine in Simulink using subtraction method. After that we have implemented on TMS320C6713 DSP processor in real-time environment. A Simulink model is linked to code composer studio through embedded target and Real Time workshop facility to generate corresponding $\mathrm{C}$ code. The generated $\mathrm{C}$ code is used for the DSP processor to perform Karaoke Machine.
\end{abstract}

\section{Keywords}

Karaoke Machine, TMS320C6713, MATLAB, Simulink

\section{INTRODUCTION}

Karaoke is a form of entertainment in which users sing along with recorded music using a microphone. So our first phase of our Karaoke machine involves removing the original voice of the artist in a song. We can establish this application based hardware model by generating a CCS project and downloading corresponding codes on TMS320c6713DSK.

MATLAB is the foundation of our project activity. In MATLAB we have designed the Simulink model of Karaoke system that can be downloaded on the kit using CCS link available in the MATLAB. Simulink is software for modeling, simulating, and analyzing dynamic systems. Various MATLAB operations and functions are used. MATLAB tools like GUI, FDA, COLEA and SP tool are also utilized. Using GUI i.e. Graphical User Interface, the user friendly environment can be provided. Using FDA Tool we can design digital filters of higher orders and observe magnitude and frequency plots.

TMS320C6713DSK kit is the hardware backbone of our project therefore this paper also provides key features, functional overview and board layout of DSK 6713 which is a low-cost standalone development platform.

\section{THE TMS320C6713 DSK}

The C6713 DSK is a low-cost standalone development platform that enables users to evaluate and develop applications for the TI C67xx DSP family. The DSK also serves as a hardware reference design for the TMS320C6713 DSK. Schematics, logic equations and application notes are available to ease hardware development and reduce time to market. Figure 1 represents the block diagram of C6713DSK kit.

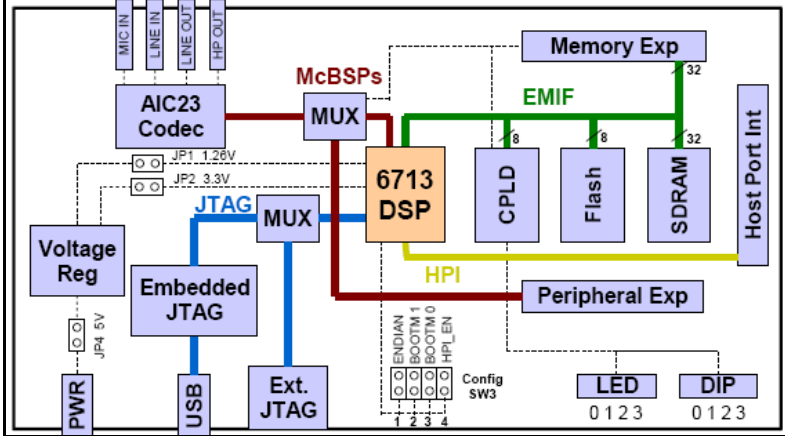

Fig 1: Block Diagram of C6713DSK

The DSK comes with a full complement of on-board devices that suit a wide variety of application environments. Key features include:

- A Texas Instruments TMS320C6713 DSP operating at 225 $\mathrm{MHz}$

- An AIC23 stereo codec

- 16 Mbytes of synchronous DRAM

- 512 Kbytes of non-volatile Flash memory (256 Kbytes usable in default configuration)

- 4 user accessible LEDs and DIP switches

- Software board configuration through registers implemented in CPLD

- Configurable boot options

- Standard expansion connectors for daughter card use

- JTAG emulation through on-board JTAG emulator with USB host interface or external emulator

- Single voltage power supply $(+5 \mathrm{~V})$

1350 million floating-point operations per second (MFLOPS), 1800 million instructions per second (MIPS), and with dual fixed-/floating-point multipliers up to 450 million multiplyaccumulate operations per second (MMACS).

The DSP on the 6713 DSK interfaces to on-board peripherals through a 32-bit wide EMIF (External Memory Interface). The SDRAM, Flash and CPLD are all connected to the bus. EMIF signals are also connected daughter card expansion connectors which are used for third party add-in boards.

The DSP interfaces to analog audio signals through an onboard AIC23 codec and four $3.5 \mathrm{~mm}$ audio jacks (microphone input, line input, line output, and headphone output). The codec can select the microphone or the line input as the active input. The analog output is driven to both the line out (fixed gain) and headphone (adjustable gain) connectors. McBSP0 is used to send commands to the codec control interface while McBSP1 is used for digital audio data. McBSP0 and McBSP1 can be re-routed to the expansion connectors in software. 
A programmable logic device called a CPLD is used to implement glue logic that ties the board components together. The CPLD has a register based user interface that lets the user configure the board by reading and writing to its registers.

The DSK includes 4 LEDs and a 4 position DIP switch as a simple way to provide the user with interactive feedback. Both are accessed by reading and writing to the CPLD registers. An included $5 \mathrm{~V}$ external power supply is used to power the board. On-board switching voltage regulators provide the $+1.26 \mathrm{~V}$ DSP core voltage and $+3.3 \mathrm{~V} \mathrm{I} / \mathrm{O}$ supplies. The board is held in reset until these supplies are within operating specifications. Code Composer communicates with the DSK through an embedded JTAG emulator with a USB host interface. The DSK can also be used with an external emulator through the external JTAG connector. Figure 2 represents the board layout of DSK6713 kit.

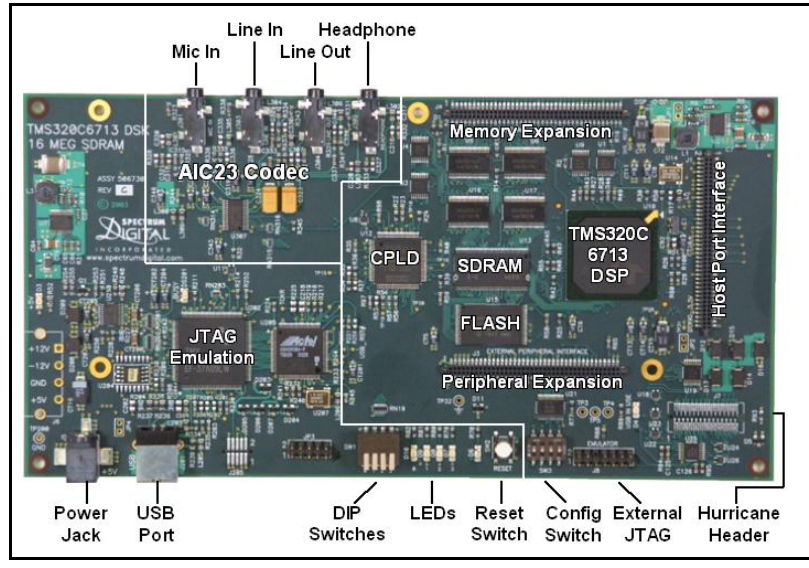

Fig 2: DSK6713 kit

\section{DESIGN PROCESS}

Please For suppression of voice, we can apply Equalizers to the original recording for attenuating the vocal frequency bands of the spectrum. For this we have to use multiple filters of varying pass band frequencies. But such filters cannot remove the vocal completely and change/distorts the pitch of the original voice. The bass component gets suppressed, distorted and the spectral composition of the original signal will also get changed.

So, to reduce voice component, we have splitted a recorded stereo sound track into two channels. This will generate two mono sound tracks (left and right). After that we have subtracted the left and right channel of the original recording. By this way satisfactory performance is obtained. Voice of the original artist is effectively reduced and can be suppressed completely if recorded music is fairly balanced. Figure 3 represents the method of original voice suppression with the help of Karaoke Machine.

We usually have two channels: left and right for recording a song in a music studio in stereo form. So, the voice of the artist standing at the center of the studio stage is simultaneously and equally fed into both these channels. This results in same vocal component in both the left and right tracks of the audio song. But when we are recording the music of multiple instruments, the left and right channels will have different musical components of the stereo sound output. By this way satisfactory performance is obtained. Voice of the original artist is effectively reduced and can be suppressed completely if recorded music is fairly balanced.

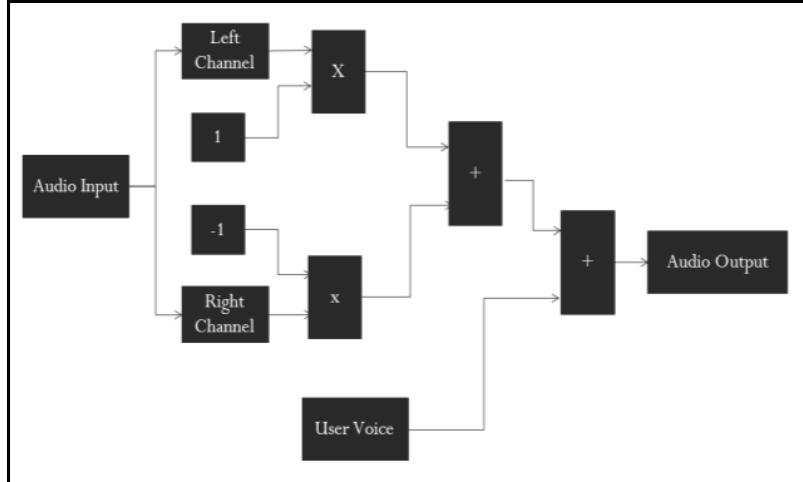

Fig 3: Block diagram of Karaoke Machine.

We have implemented this method using Simulink as shown in figure no 4. In simulink model audio file is read and then splitted into two parts-left and right channel. 1 is multiplied to left channel and -1 is multiplied to right channel. So left channel will remain as it and right channel will we inverted.After that both the channels are added.This results in the removal of same component and presence of different component in the output.

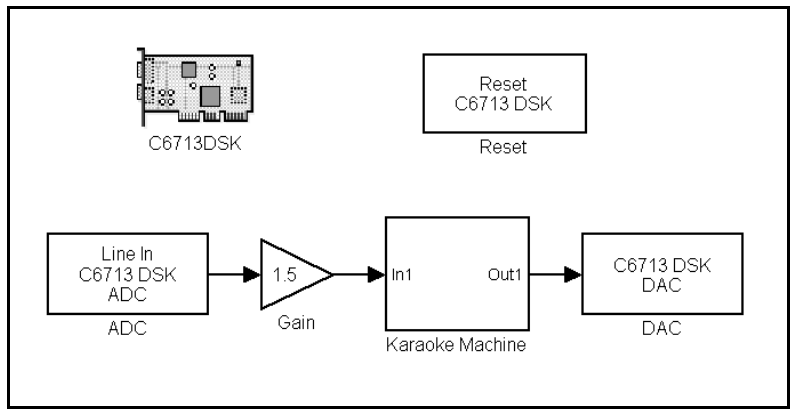

Fig 4: Simulink Model for Implementation of Karaoke Machine

We have implemented our method with simulink, but it is not possible to load our simulink model directly to DSK6713 processor. The DSP development software, Code Composer Studio can accept either C or assembly code to generate output (.out) file, which can be load on DSP chip. The programming in $\mathrm{C}$ or assembly is not easy for every researcher. Thus the designed Simulink model facilitates to generate $\mathrm{C}$ code automatically corresponding to the desired problem with the help of embedded target and RTW facility provided in Simulink. . So,we are using Code composer studio to generate .out file. This file now we can load into our DSK6713 processor. Figure 5 represents the interfacing between MATLAB, CCS and DSP.

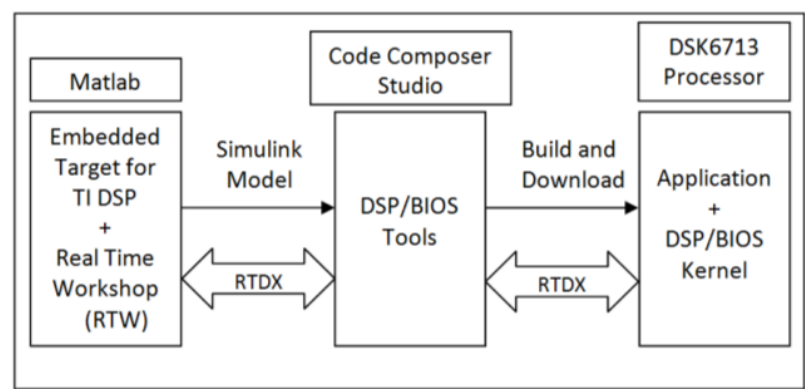

Fig 5: Interfacing between MATLAB, CCS and DSP.

Figure 6 represent the process of basic downloading in the form of flowchart. 


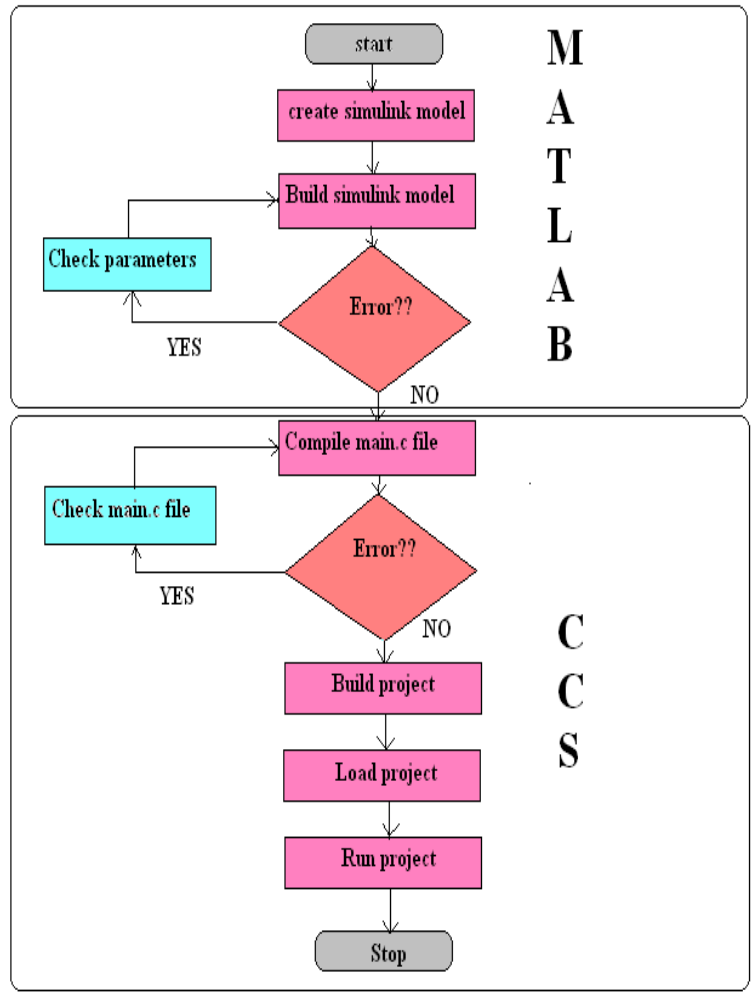

Fig 6: Flowchart of basic downloading process.

\section{SIMULATION RESULTS}

After simulation of an original song file in simulink, we get following results. Karaoke Machine output and Original song output is shown in figure no 7. In this graph we can observed that due to the effect of reduction or suppression of original voice, some portion of speech is suppressed.

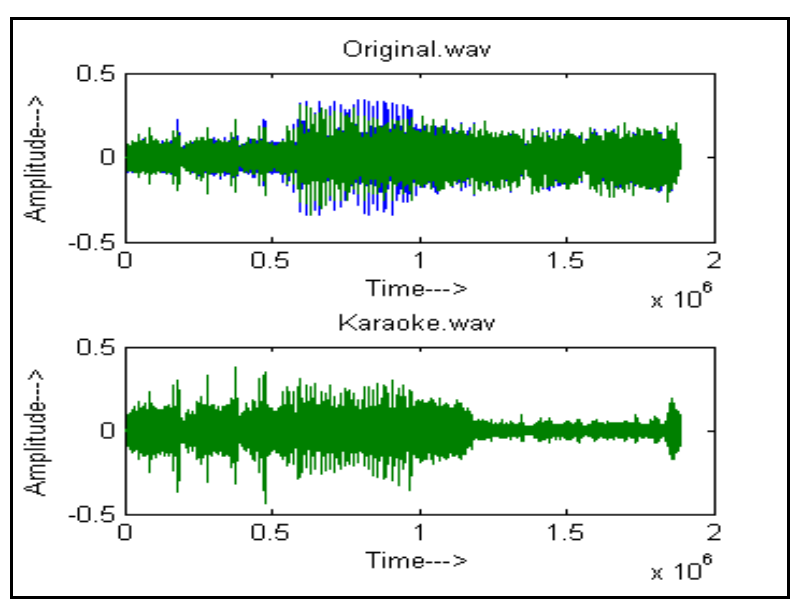

Fig 7: Original song and Karaoke song

Figure 8 and Figure 9 represents the output of original song and Karaoke song from DSK6713.

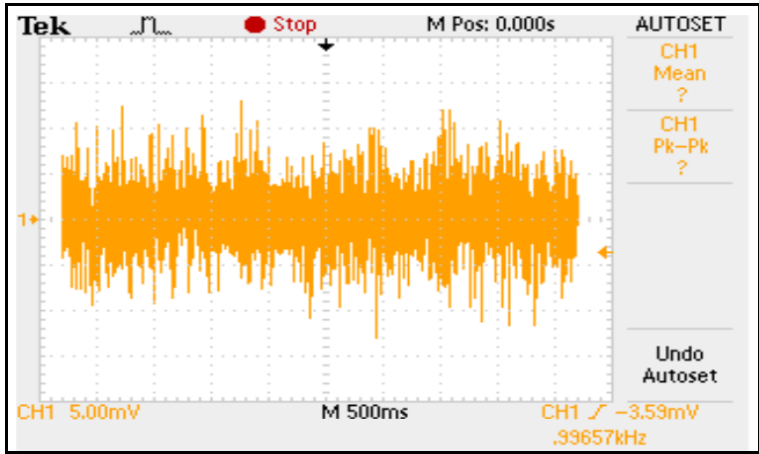

Fig 8: Output of Original Song from DSK6713

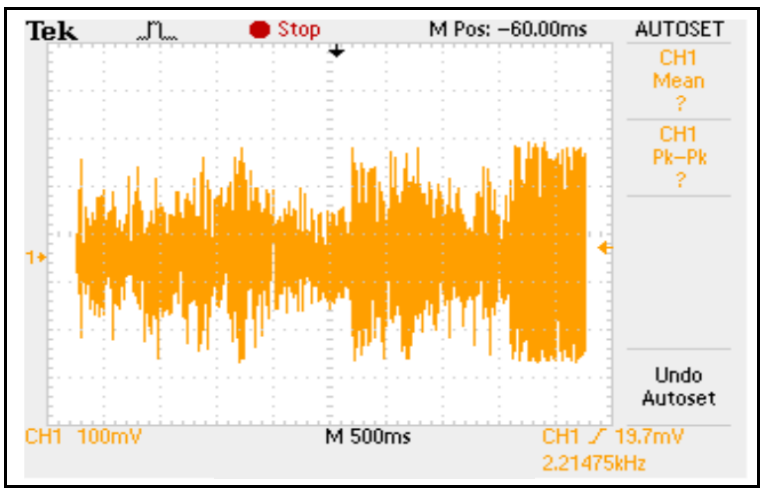

Fig 9: Output of Karaoke Song

\section{CONCLUSION}

So through the medium of this research paper we have tried to present a new approach for removing or suppressing original artist's voice from an original song. Further we have added some stages like amplifier, bass booster and treble booster using Dip switch available on the DSK.

Also we conclude that Digital signal processors such as the TMS320C6x family of processors are like fast specialpurpose microprocessors with a specialized type of architecture and an instruction set appropriate for signal processing. The C6713 DSK allows you to download and step through code quickly and uses Real Time Data Exchange for improved Host and Target communications. The DSK includes the Fast Run Time Support libraries and utilities such as Flash burn to program flash, Update Advisor to download tools, utilities and software and a power on self test and diagnostic utility to ensure the DSK is operating correctly.

\section{REFERENCES}

[1] Jihoon Park, Jungpyo Hong, Kwangki Kim, and Minsoo Hahn "Harmonic elimination structures for Karaoke Mode in Spatial Audio Object Coding Scheme", ICCE conference, published in 2011

[2] Wei-Ho Tsai , Hsin-Chieh Lee "An Automated Singing Evaluation Method For Karaoke Systems", ICASSP conferace 2011

[3] Dolly Reney, Dr.Neeta Tripathi "Signal Generation Using TMS320C6713 Processor” IJCSET, December 2011, Vol 1, Issue 11, 753-756, ISSN-2231-011

[4] Raj Kumar Thenua, S. K. Agrawal, Member, "Hardware Implementation of Adaptive Algorithms for Noise Cancellation" IACSIT, Vol. 2, No. 2, March 2012

[5] Udo Zoelzer (Ed). DAFX:Digital Audio Effects. J. Wiley \& Sons, Chichester, 2002 